\title{
Short-Term Forecasting of Solar Irradiance using STL, Wavelet and LSTM
}

\author{
Vivek Vijay \\ Dept of Mathematics \\ IIT Jodhpur \\ Jodhpur, India
}

\author{
Rakesh Kumar \\ Dept of Mathematics \\ IIT Jodhpur \\ Jodhpur, India
}

\author{
Ashish Sharma \\ Dept of Mathematics \\ IIT Jodhpur \\ Jodhpur, India
}

\author{
Abhishek Kumar \\ Dept of Mathematics \\ IIT Jodhpur \\ Jodhpur, India
}

\begin{abstract}
We propose a hybrid framework for short-term forecasting based on decomposition techniques and LSTM (Large Short Term Memory) algorithm. The study aims to analyze and quantify solar irradiance forecast using two types of decomposition techniques: decomposition of time series into the time domain and frequency domain via locally weighted regression based on Loess (STL) and using discrete wavelet transformation (DWT) respectively. LSTM is used to forecast the decomposed detail and approximation components. The final forecast of GHI (Global Horizontal Irradiance) is then obtained by combining these components using inverse wavelet transform. Hourly data from the Indian Meteorological Department (IMD) Jodhpur Rajasthan from January 2017 to June 2019 is used to demonstrate the proposed algorithm. The forecast accuracy of the proposed model is compared with other competitive models. The observed results show that the proposed combination of STL, wavelet transform, and LSTM outperforms (1) LSTM, (2) combination of STL and LSTM, (3) Bi-LSTM and (4) the persistence model.
\end{abstract}

\section{Keywords}

Forecasting, Ensemble Forecast, renewable energy, wavelet transform

\section{INTRODUCTION}

The wind and solar power developments have been substantial in recent years. Presently, the contribution of solar PV is more than that of CSP because the latter is capital intensive and has a high levelized cost of electricity(LCOE) [4]. Although integration of CSP into power system is less challenging due to its thermal aspects, which reduce variability in output. The variability is one of the biggest challenges for generation owners and grid operators in integrating solar generation. Challenges are due to the intermittent and unpredictable nature of renewable energy resources which intensify the complexity of grid management while maintaining the demand/ supply balance [26, 2]. This leads to some other problems, such as voltage fluctuations, local power quality, and stability issues. Therefore, solar irradiance forecasting becomes crucial for solar installation operators, electricity market operators and electricity supply infrastructure managers. It also helps in estimating the reserves, scheduling the power system, congestion management, op- timal management of storage for trading the produced power in the electricity market, and finally to achieve a reduction in the cost of electricity production [2, 24, 32, 18]. Short-term forecast of solar resources is particularly useful for power plant operations, grid balancing, real-time unit dispatching, and energy trading [26].

The data-driven methods of forecasting have become very popular due to the easy availability of historical data and advancement of computational techniques. Nowadays, more emphasis is given to the development of ensemble methods which improve forecast accuracy and eliminate limitations of stand alone models. These ensemble forecasting methods are primarily divided into competitive ensemble forecasting and cooperative ensemble forecasting [23]. The second one is further categorized into pre-processing and postprocessing ensemble forecasting. [25].

We propose a hybrid model which combines STL (Seasonal and Trend Decomposition using Loess), wavelet transform, and long short term memory (LSTM) to forecast the hourly global horizontal irradiance. STL is used to separate seasonal variability from the data. STL decomposes the data into trend, seasonal variation, and irregularity (residual) components. Wavelet transform is then applied on the sum of trend and residual components. The output of wavelet transform is fed as an input to the LSTM model. The forecast obtained by LSTM is added to the seasonal variation to get the final forecast value.

\subsection{Related work}

In this subsection, we discuss some of the recent studies published on solar forecasting using hybrid but data driven models. Hybrid models based on wavelet transform and artificial neural network (ANN) are discussed in [6, 28, 22]. Hybrid of support vector machine (SVM) and discrete wavelet transform (DWT) was introduced by Mohammadi et al. to predict horizontal global solar radiation [21]. Among all the wavelet-based hybrid models, DWT was used to decompose the time series into several sub-series with more detailed periodic information, which is easy to forecast. Time-delay neural networks (TDNN) along with autoregressive moving average (ARMA) were proposed by [16]. An ARMA model was used to model the linear pattern present in the data, followed by artificial neural networks (ANN) to fit the non-linear pattern. Another hybrid of an evolutionary algorithm with Support Vector Machine (SVM) and ANN was proposed in the two articles [11 12]. Silisiri- 
vanich [29] analyzed the solar irradiance data using wavelet transform. The study decomposed global irradiance components into direct and indirect ones. The objective was to see the decomposition of data using wavelet transformation. A prediction model of rolling type, consisting of empirical mode decomposition (EMD) and an ANN, to predict solar radiation of Gonghe county of China was proposed by [13]. In this model, they considered only simple solar radiation data instead of topographic meteorological data. Huang et al.[15] used a hybrid wavelet transformation and Elman model to forecast hourly global irradiation. This model produced better results across all the seasons. In this model, decomposition of solar irradiance was done using wavelet transformation and the Elman neural network was used for forecasting every sub-series. This model outperformed many other methods and obtained 0.7590 average forecast skill over other models. Evolutionary algorithms like self-organizing maps (SOM) was applied to partition the input datasets into several disjoint sub-datasets and particle swam optimization (PSO) was implemented to select the parameters. Finally, used an appropriate predictor like ANN or SVM. Recently, several hybrid frameworks for hourly solar irradiance forecast, based on different data mining techniques, were reported in [3] 14, 17]. In all these data mining-based hybrid frameworks, clustering techniques were prominently used. A proper cluster selection algorithm was generally used to select the most suitable clusters. The appropriate cluster data was then modeled using one of the predictors, such as ANN or SVM. They also worked and proposed another famous decomposition technique called seasonal and trend decomposition using Loess (STL) [31, 33]. Alzahrani et al.[1] introduced a new technique for forecasting solar irradiance using Deep Recurrent Neural Network (RNN). This work improved the model complexity and helped in extracting high-level features. Performance was compared with ANN and SVM. The architecture of RNN[10] is a type of sequence model different from neural networks. The hidden layer neurons of an RNN are connected and share the information from previous inputs. RNN architecture has problems of vanishing gradient and exploding gradient. New variations of RNN resolved this problem, such as Long Short-Term Memory (LSTM) network[27], Bi-directional LSTM networks and Gated Recurrent Units (GRU). These new variants of RNN perform better than the traditional perceptron architectures, especially for the forecasting of solar irradiance time series data [30]. Brahma and Vadhwani [5] used LSTM and Bi-directional LSTM for one day ahead solar forecasting.

We present a combination of STL, wavelet transform and LSTM, which outperforms the existing models. The paper is organized as follows:

Section-2 offers forecasting tools starting from STL decomposition, wavelet transform, and long short term memory (LSTM) network. The proposed algorithm, along with the analysis of data, is presented in Section-3. Metrics for performance evaluation are given in Section-4. Section-5 contains results and conclusions.

\section{FORECASTING TOOLS}

\subsection{STL decomposition}

STL is a filtering procedure employed for the additive decomposition of a time series into its three constituent components; seasonal, trend, and remainder [7]. STL decomposes a time series in the time domain without using any deterministic function like the mother wavelet in wavelet decomposition. STL filters the data through a sequence of applications of the Loess smoother and moving aver- age [8].

The complete STL procedure is an iteration cycle of de-trending by using Loess, local regression polynomial fitting, and then updating the seasonal component at every iteration. The robustness weights are estimated based on irregular features, which make the model robust to outliers. The iterative cycle consists of two recursive procedures, the inner and the outer loops. Each pass of the inner loop applies seasonal smoothing followed by trend smoothing that updates the seasonal and trend components, respectively. An iteration of the outer loop consists of one iteration of the inner loop to estimate trend and seasonal components, which are further used for the calculation of irregularity components. For a more detailed description of the STL decomposition procedure and Loess polynomial fitting, see [7, 8].

\subsection{Discrete wavelet transform (DWT)}

The wavelet transform possesses good time and frequency resolution simultaneously by using short windows at high frequency and long windows at low frequency. Effective applications of wavelet heavily depend on the choice of wavelet family, mother wavelet, and its order. DWT uses successive high pass and low pass filtering operations to decompose the signals into approximations and detailed series for further analysis. The DWT utilizes two sets of functions, the scaling function $\phi(t)$ and the wavelet function $w(t)$. The scaling function $\phi(t)$ is associated with the low pass filters with filter coefficients $b(n), n \in Z$ and the wavelet function $w(t)$ with the high-pass filters with filter coefficients $c(n), n \in Z$, respectively. According to Mallat's algorithm [19], DWT is realized by means of the filters $b[k], c[k]$ that are related to each other as

$$
c[k]=(-1)^{k+1} b[N-k-1], \text { for } k=0,1, \ldots, N-1,
$$

where $N$ is the length of the filter. These filters are constructed from the scaling function $\phi(t)$ and the wavelet function $w(t)$ as,

$$
\begin{aligned}
& \phi(t)=\sqrt{2} \sum_{k} b[k] \phi(2 t-k), \\
& w(t)=\sqrt{2} \sum_{k} c[k] \phi(2 t-k) .
\end{aligned}
$$

Outputs of these filters are sets of low and high frequency components named as approximations $a_{j}[k]$ and details $d_{j}[k]$ respectively and, are defined by,

$$
\begin{aligned}
& d_{j+1}(k)=\sum_{n} a_{j}[n] c(2 k-n), \\
& a_{j+1}(k)=\sum_{n} a_{j}[n] b(2 k-n),
\end{aligned}
$$

here, $a_{j}[k]$ represents the tendency of the series which is characterized by slow dynamics (less variability), while the details $d_{j}[k]$ present the local details of time series and have fast dynamics (high variability).

\subsection{Recurrent Neural Network (RNN)}

An RNN is an extension of neural network structure, which is proposed to see that "human cognition is based on the memory and its encounters." It is different from the Deep Neural Network and 


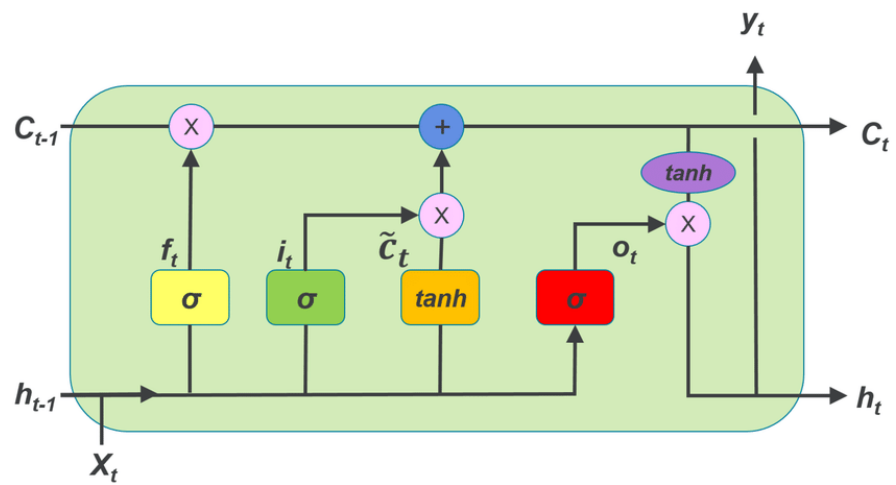

Fig. 1. Architecture of LSTM

Convolutional Neural Network as these architectures do not depend on their past inputs and give the arrangement of a memory work of the past content. RNN remembers the past data and applies it to the current output. It is a sequence model, the output of the hidden layer depends on the input from that hidden layer and the input from the previous hidden layers.

2.3.1 Long Short-Term Memory (LSTM). LSTMs, special cases of RNNs, are capable of learning long-term dependencies. An LSTM architecture has a recurrence of cells instead of hidden layers used in conventional neural networks. The cell in the LSTM network consists of input gate, output gate, forget gate, and cell state. The cell structure consists of an activation functions such as sigmoid function or tanh function. Operations of different gates are stated below:

(1) Input gate: It acts as an input to the cell. The input to this gate is $x_{t}$ that represents the present input to the cell and $h_{t-1}$, the previous output of the cell.

(2) Cell state: It is used for either retaining or removing of information with the help of gates.

(3) Forget gate: It decides which part of the information is relevant and accordingly allowed to update the information stored in memory.

(4) Output gate: It is the output part of the cell.

(5) Sigmoid layer: The output of the sigmoid activation function ranges from 0 to 1 . This activation function is used to produce a normalised value or a probability value as output.

(6) Tanh layer: This layer is used to generate a new vector which is to be added to the cell state. The mathematical structure of an LSTM is given below -

$$
\begin{gathered}
f_{t}=\sigma\left(W_{f}\left[h_{t-1} * x_{t}\right]+b_{f}\right) \\
i_{t}=\sigma\left(W_{i}\left[h_{t-1} * x_{t}\right]+b_{i}\right) \\
C_{t}=\tanh \left(W_{c}\left[h_{t-1} * x_{t}\right]+b_{c}\right) \\
O_{t}=\left(W_{o}\left[h_{t-1} * x_{t}\right]+b_{o}\right) \\
h_{t}=O_{t} * \tanh \left(C_{t}\right)
\end{gathered}
$$

where $x_{t}$ is the input vector, $h_{t}$ is output vector, $C_{t}$ is the cell state vector, $f_{t}$ is forget gate vector, $i_{t}$ is input gate vector, $O_{t}$ is output gate vector and, $W$ and $b$ are the parameter matrix and vector, respectively.
The two inputs $x_{t}$, the present input, and $h_{t-1}$, the previous output, pass through the sigmoid activation function (denoted by $\sigma$ ) to generate the output $f_{t}$. This gate is called a forget gate. The following step is to choose unused data that needs to be stored in the cell state. To begin with, a sigmoid layer called "the input gate layer" determines the values to be upgraded. The other one, a tanh layer makes a vector of new candidate values, $C_{t}$, that is included in the state. Within this second step, we combine these two for further upgradation. The next step is to upgrade the cell state $C_{t-1}$ as we need to integrate new information with it. The forget gate value $f_{t}$ is now multiplied with $C_{t-1}$. Here, $f_{t}$ represents the old state, achieved by omitting some of the earlier less relevant information. After this, a term $i_{t} * C_{t}$ is added with $f_{t} * C_{t-1}$ to get the required updated cell state. Next, the cell state is passed through the tanh function (this normalises the output from -1 to 1 ) and is multiplied with the output of the forget gate which gives the output of the complete cell, $h_{t}$. An architecture of LSTM is presented in Figure 1

\section{PROPOSED METHODOLOGY}

The objective of this work is to present a hybrid forecasting framework for short-term forecasting of solar data. It separates the trend and irregularity components from the seasonal variation and then uses LSTM to forecast each of these decomposed components. STL filtering process allows us to decompose solar irradiance data into a deterministic component (seasonal) and a random component (residual). The flow diagram of the whole procedure is shown in Figure 2

The proposed forecast method can be summarized in the following step by step algorithm:

(1) Decompose the original GHI series $\left(X_{t}\right)$ into seasonal $\left(s_{t}\right)$, trend $\left(m_{t}\right)$ and remainder series $\left(e_{t}\right)$ using STL decomposition.

(2) Subtract the seasonal component $\left(s_{t}\right)$ from the original series $\left(X_{t}\right)$ to get residual series $\left(R_{t}\right)$ which is actually the sum of trend $\left(m_{t}\right)$ and remainder component $\left(e_{t}\right)$.

(3) The residual series is then normalized by using,

$$
Y_{t}=\frac{R_{t}-\min \left(R_{t}\right)}{\max \left(R_{t}\right)+\min \left(R_{t}\right)}
$$

(4) The normalized residual series $Y_{t}$ is then decomposed using three time-steps Mallat's decomposition algorithm of wavelet decomposition. This results in a low frequency sequence $A(t)$ and a high-frequency sequences $d_{1}(t), d_{2}(t), d_{3}(t)$.

(5) The above sequences $\mathrm{A}(\mathrm{t}), d_{1}(t), d_{2}(t)$, and $d_{3}(t)$ are used for forecasting using LSTM.

(6) Inverse wavelet is used to combine the forecast values $\hat{A}(t+1)$, $\hat{d}_{1}(t+1), \hat{d}_{2}(t+1), \hat{d}_{3}(t+1)$ to obtain $\hat{Y}_{t+1}$.

(7) The data series $\hat{Y}_{t+1}$ is de-normalized using,

$$
\hat{R}_{t+1}=\hat{Y}_{t+1} *\left(\max \left(R_{t}\right)+\min \left(R_{t}\right)+\min \left(R_{t}\right),\right.
$$

where, $\hat{R}_{t+1}$ denotes the de-normalized residual data series after forecasting.

(8) The forecast data series $\hat{R}_{t+1}$ is added to the seasonal component of data series to obtain the final forecast $\hat{X}_{t+1}$. 


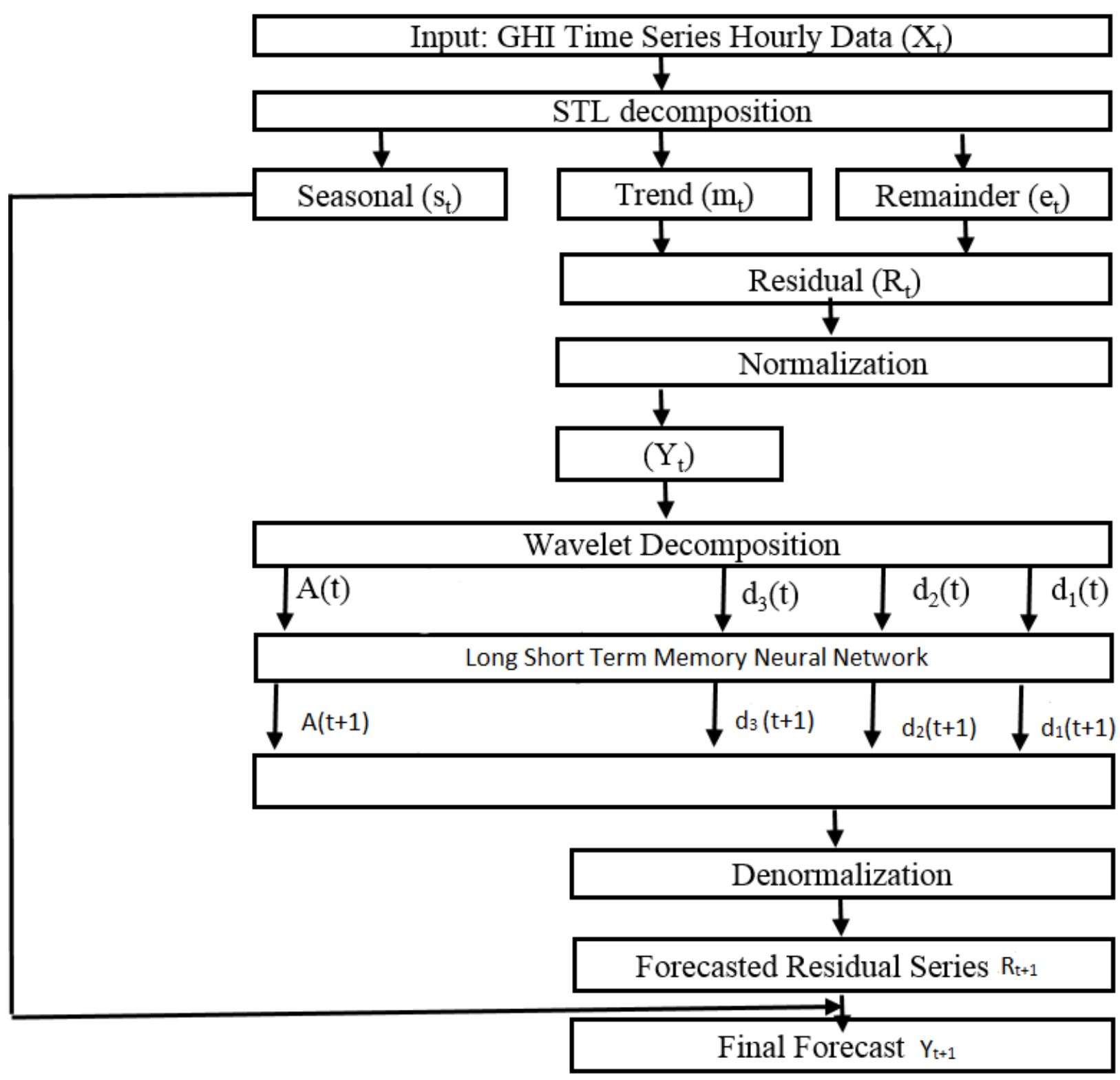

Fig. 2. Flowchart of the proposed methodology

\subsection{Analysis of Data}

The data is hourly records of global horizontal irradiance (GHI) collected by Indian Meteorological Department (IMD) Jodhpur, India from January 1, 2017 to June 30, 2019 (911 days). The analysis is carried out for the $12^{\text {th }}$ hour. To demonstrate the validity of the proposed methodology, one day ahead forecast of solar irradiance for the $12^{\text {th }}$ hour, using STL along with wavelet analysis and LSTM is carried out. Using STL decomposition, the data is decomposed into seasonal, trend, and remainder data sub-series. Figure 3 shows the data series and the decomposed sub-series of the $12^{t h}$ hour. After decomposition of the data, the residual series is obtained by subtracting the seasonal sub-series from the data.
Now the residual sub-series of the data is further decomposed using Mallet's pyramidal method with the mother wavelet of 'coif5'. The low-frequency sequence $A(t)$ and the high-frequency sequences $d_{3}(t), d_{2}(t)$, and $d_{1}(t)$ of each month solar irradiance are obtained by the 3 -scale wavelet decomposition. The algebraic sum of the low-frequency sequence and the high-frequency sequences constitutes the original data sequence. Then, $A(t)$ and $d_{3}(t), d_{2}(t), d_{1}(t)$ are used as inputs to the network. The network training function is used to update weights and bias values in 'trainlm', which follow Levenberg-Marquardt optimization procedure. A 3-layer LSTM is constructed in the following way. January to June data is used to train the model. After that, the previous 30 day's data is used to predict the data for the 31 st day. For example, to predict $1^{\text {st }}$ September 

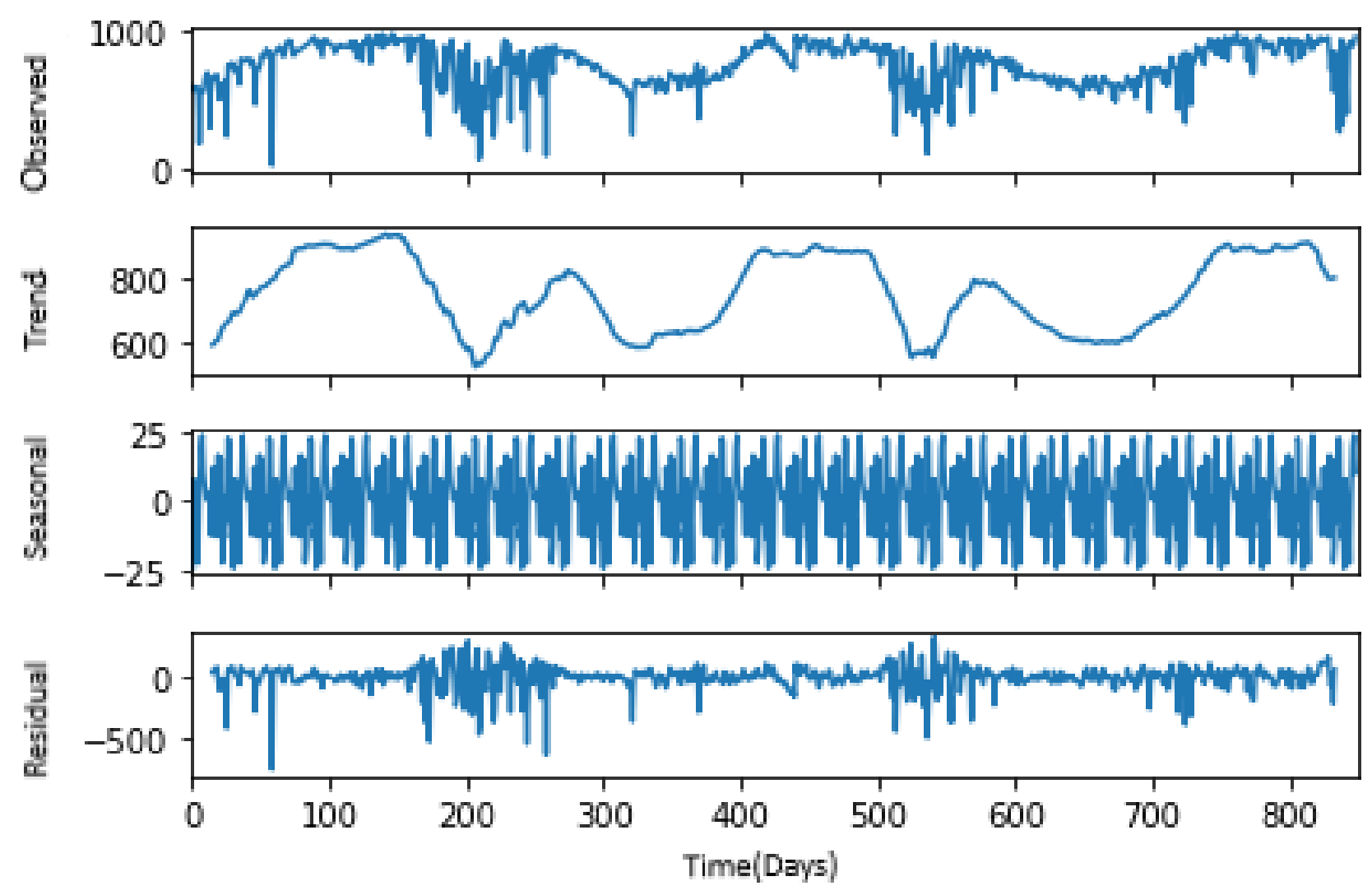

Fig. 3. STL decomposition of the time series of $12^{\text {th }}$ hour

value, the data of August is used. For predicting the next day value, one day shift was used in feeding the data. Figure 4 shows the actual wavelet decomposed sub-series and its corresponding forecast sub-series.

\section{PERFORMANCE EVALUATION METRICS}

Forecast accuracy depends on weather conditions and forecast's temporal/ spatial resolution. A number of metrics for performance evaluation are listed in the literature [2, 34, ?]. However, RMSE (Root Mean Square Error) and MAE (Mean Absolute Error) metrics are most commonly used to evaluate forecast error. Standard error of forecasting is calculated by the RMSE and the MAE as

$$
\begin{array}{r}
R M S E=\sqrt{\frac{\sum_{t=1}^{n}\left(X_{t}-\hat{X}_{t}\right)^{2}}{n},} \\
M A E=\frac{\sum_{t=1}^{n}\left|X_{t}-\hat{X}_{t}\right|}{n} .
\end{array}
$$

Forecast accuracies are not comparable site-by-site unless normalized by a benchmark. The forecast skill (S) is a way to normalize forecast accuracy [9, 20].

$$
S=1-R M S E m / R M S E p
$$

where RMSEm is the RMSE value of the model under consideration and RMSEp is the RMSE value of the persistence model. As per the definition of the forecast skill, for perfect solar forecast $S=1$, and if $S=0$, the forecast uncertainty is as significant as variability. By definition, for average model $S=0$.

\section{RESULTS}

Forecast error and forecasting skill of each monthare presented in Table ?? and 2 respectively. The $12^{\text {th }}$ hour data series of first day of all months and its forecast using (i) LSTM, (ii)BiLSTM,(iii) STL along with LSTM and (iv) STL, wavelet along with LSTM are shown in the Figure 5

A new irradiance forecasting model based on STL combined with the wavelet and LSTM technique is presented in this work. Basic idea of the proposed model is two-stage decomposition of data, using STL and wavelet decomposition techniques to use advantages of the combined model. STL decomposition along with wavelet decomposition of data helps in reducing the complexity of the data in the more prominent way and enhance the learning of LSTM.

To show the effectiveness of the proposed model, it is compared with the persistence model, LSTM and SLSTM (LSTM with STL). The persistence model is a standard benchmark model in which the forecasted value is given by the current value of the time series. RMSE and MAE values of each month for the proposed model are significantly low compared to the other models. The calcu- 

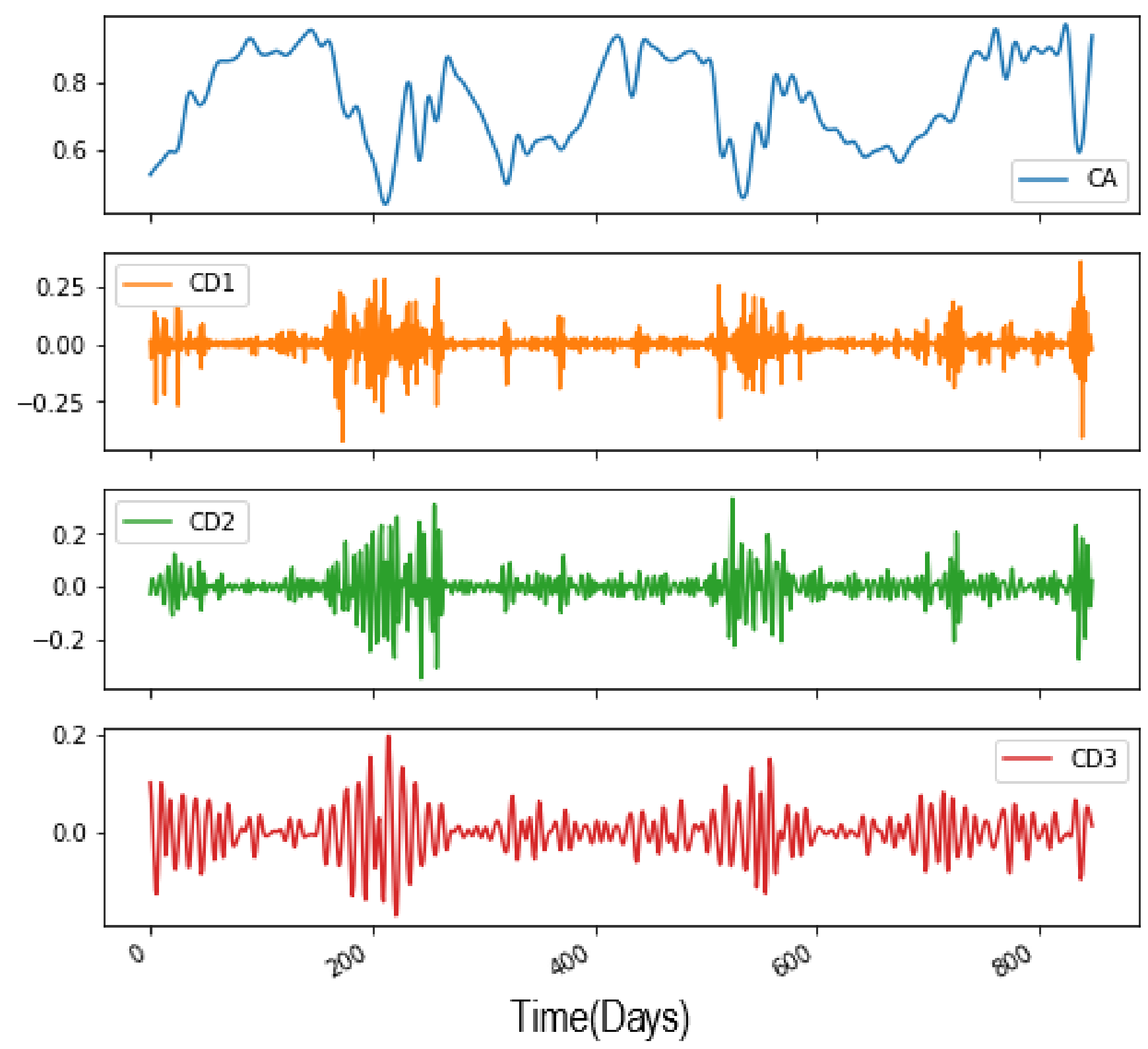

Fig. 4. Actual wavelet decomposed sub-series $\left(A(t), d_{1}(t), d_{2}(t), d_{3}(t)\right.$ of $12^{t h}$ hour

lated RMSE and MAE values are high for LSTM and gradually decreases for SLSTM, and are minimum for SWLSTM (LSTM with STL and wavelet) for all the months. Moreover, the forecast skill also shows the improvement in its value for the proposed hybrid model, given in Table 2 which further strengthens our claim. Additionally, we use a statistical test (one-tailed t-test) to test whether the RMSE of SWLSTM is significantly lesser than the RMSE of SLSTM, LSTM, and persistence model. We have performed one tail t-test over the RMSE value of SWLSTM against the RMSE value of SLSTM and calculated the p-value. This clearly shows that the reduction in the error is significant. Similarly, we perform the t-test over RMSE value of SLSTM and LSTM and calculate the p-value. Likewise, we calculate the p-value between RMSE of SWLSTM and persistence model. This shows that the hypothesis of no reduction is rejected for all three cases.

\section{CONCLUSIONS}

In this work, we combine LSTM algorithm with STL and wavelet transform to develop a new approach, SWLSTM, for forecasting of global horizontal irradiation. STL decomposition was used to get three constituents (trend, residual and seasonality) and further wavelet analysis was used to get approximation and detail coefficients. These are utilised as input for LSTM to get the final fore- 


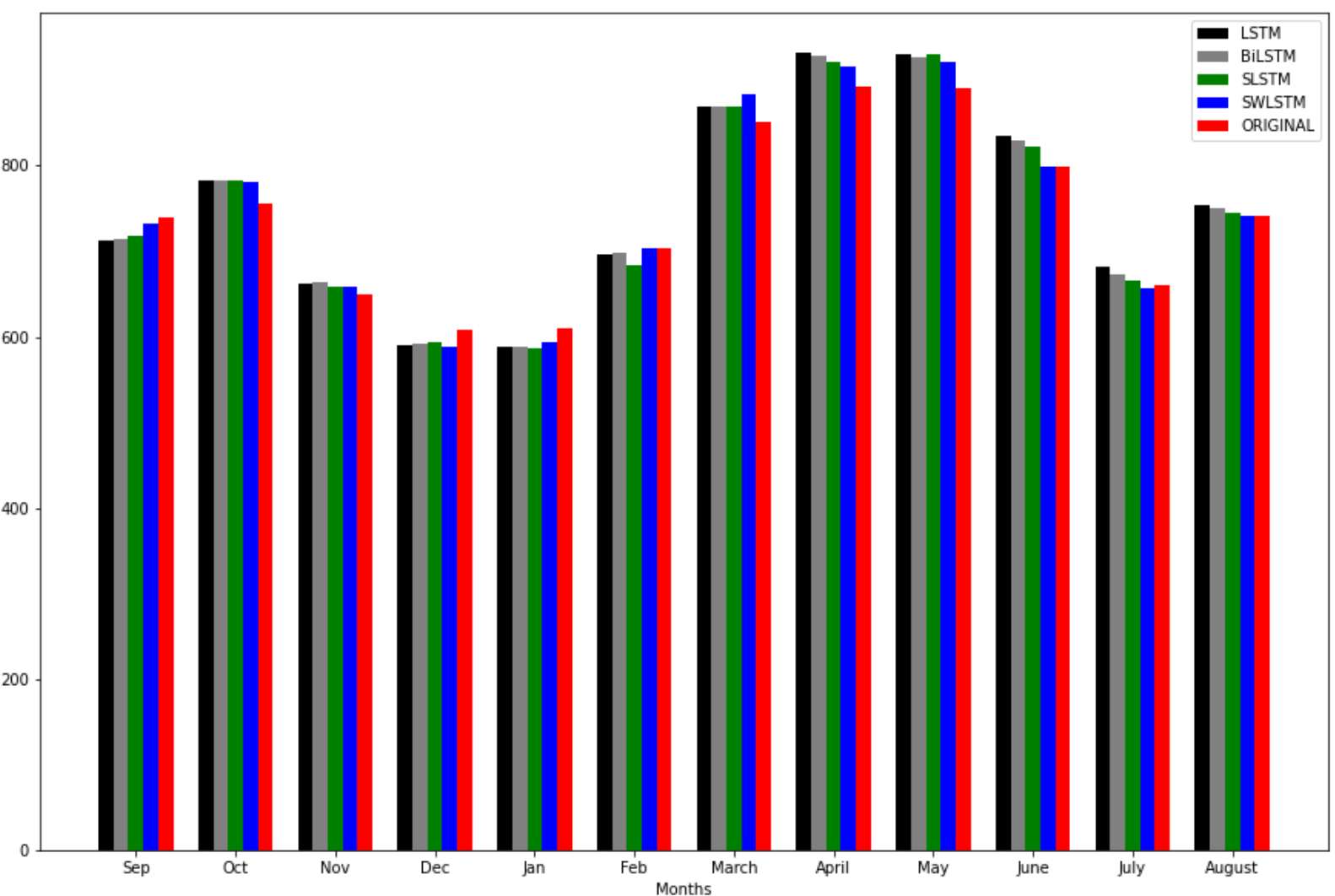

Fig. 5. Comparison between average of original and predicted values of all month's GHI using different models $\left(W / m^{2}\right)$

Table 1. Error comparison table

\begin{tabular}{|c|c|c|c|c|c|c|c|c|c|c|}
\hline \multirow{2}{*}{$\begin{array}{l}\text { Training } \\
\text { Data } \\
\end{array}$} & \multicolumn{2}{|c|}{ Persistence } & \multicolumn{2}{|c|}{ LSTM } & \multicolumn{2}{|c|}{ BiLSTM } & \multicolumn{2}{|c|}{ SLSTM } & \multicolumn{2}{|c|}{ SWLSTM } \\
\hline & MAE & RMSE & MAE & RMSE & MAE & RMSE & MAE & RMSE & MAE & RMSE \\
\hline September & 124.14 & 204.32 & 129.91 & 169.29 & 121.33 & 159.71 & 110.32 & 149.21 & 44.68 & 53.04 \\
\hline October & 39.92 & 62.47 & 44.30 & 66.81 & 41.03 & 65.38 & 43.84 & 64.15 & 36.29 & 49.71 \\
\hline November & 3.19 & 4.56 & 19.34 & 33.91 & 19.14 & 30.73 & 19.98 & 29.41 & 18.34 & 25.01 \\
\hline December & 17.79 & 27.23 & 29.66 & 36.84 & 27.97 & 33.43 & 28.25 & 35.19 & 23.01 & 28.35 \\
\hline January & 51.69 & 91.95 & 58.73 & 74.52 & 56.35 & 71.80 & 45.79 & 64.21 & 28.36 & 41.73 \\
\hline February & 95.81 & 168.23 & 89.92 & 123.01 & 84.83 & 117.58 & 86.82 & 115.07 & 37.60 & 55.73 \\
\hline March & 63.49 & 110.51 & 42.36 & 77.31 & 40.27 & 72.94 & 46.19 & 76.95 & 31.05 & 51.74 \\
\hline April & 40.69 & 69.72 & 64.46 & 84.83 & 62.67 & 83.39 & 66.07 & 85.51 & 60.57 & 73.96 \\
\hline May & 51.99 & 73.14 & 48.87 & 66.29 & 47.84 & 65.73 & 53.98 & 69.52 & 49.47 & 62.12 \\
\hline June & 151.09 & 251.52 & 140.84 & 204.66 & 136.90 & 199.41 & 144.45 & 203.37 & 66.64 & 93.26 \\
\hline July & 116.67 & 213.72 & 99.63 & 178.88 & 91.39 & 167.21 & 95.51 & 175.60 & 61.37 & 89.15 \\
\hline August & 87.43 & 171.81 & 74.24 & 143.62 & 73.89 & 137.93 & 73.38 & 139.04 & 58.18 & 79.36 \\
\hline
\end{tabular}

cast. The proposed model was compared with four other models, persistence, LSTM, Bi-LSTM and SLSTM. Using the evaluation metrics MAE, RMSE and forecast skill, it was observed that the proposed model performed better and gave more accurate results on the hourly GHI forecast. It also re-establishes the fact that preprocessing significantly reduces the complexity of the data and im- 
Table 2. Forecast skill comparison table for different models

\begin{tabular}{lcc}
$\begin{array}{l}\text { Training } \\
\text { Data }\end{array}$ & $\begin{array}{c}\text { Average Model } \\
\text { (PERSISTENCE+LSTM+BiLSTM+SLTSM) }\end{array}$ & SWLSTM \\
\hline September & 0 & $\mathbf{0 . 6 9}$ \\
October & 0 & $\mathbf{0 . 2 3}$ \\
November & 0 & $\mathbf{- 0 . 0 1}$ \\
December & 0 & $\mathbf{0 . 1 4}$ \\
January & 0 & $\mathbf{0 . 4 4}$ \\
February & 0 & $\mathbf{0 . 5 7}$ \\
March & 0 & $\mathbf{0 . 3 8}$ \\
April & 0 & $\mathbf{0 . 0 8}$ \\
May & 0 & $\mathbf{0 . 0 9}$ \\
June & 0 & $\mathbf{0 . 5 6}$ \\
July & 0 & $\mathbf{0 . 5 1}$ \\
August & 0 & $\mathbf{0 . 4 6}$ \\
\hline
\end{tabular}

Table 3. p-values for the hypotheses of equality of means of RMSE for different models against SWLSTM

\begin{tabular}{lccc} 
Training & Persistence & LSTM & SLSTM \\
\hline p-value & 0.01822483 & 0.02572935 & $\mathbf{0 . 0 1 1 1 3 2 4 7}$
\end{tabular}

proves the forecast accuracy by enabling us to handle a group of data separately.

\section{ACKNOWLEDGEMENTS}

The authors would like to thank the Indian Meteorological Department (IMD) for providing the data used in this work.

\section{REFERENCES}

[1] A. Alzahrani, P. Shamsi, M. Ferdowsi, and C. Dagli. Solar irradiance forecasting using deep recurrent neural networks. pages 988-994, 2017.

[2] J Antonanzas, N Osorio, R Escobar, R Urraca, FJ Martinezde Pison, and F Antonanzas-Torres. Review of photovoltaic power forecasting. Solar Energy, 136:78-111, 2016.

[3] R Azimi, M Ghayekhloo, and M Ghofrani. A hybrid method based on a new clustering technique and multilayer perceptron neural networks for hourly solar radiation forecasting. Energy Conversion and Management, 118:331-344, 2016.

[4] Jay Prakash Bijarniya, K Sudhakar, and Prashant Baredar. Concentrated solar power technology in india: A review. $R e$ newable and Sustainable Energy Reviews, 63:593-603, 2016.

[5] Banalaxmi Brahma and Rajesh Wadhvani. Solar irradiance forecasting based on deep learning methodologies and multisite data. Symmetry, 12(11), 2020.

[6] JCSH Cao Cao and SH Cao. Study of forecasting solar irradiance using neural networks with preprocessing sample data by wavelet analysis. Energy, 31(15):3435-3445, 2006.

[7] Robert B Cleveland, William S Cleveland, Jean E McRae, and Irma Terpenning. Stl: A seasonal-trend decomposition procedure based on loess. Journal of Official Statistics, 6(1):3-73, 1990.

[8] William S Cleveland and Susan J Devlin. Locally weighted regression: an approach to regression analysis by local fitting. Journal of the American statistical association, 83(403):596610, 1988.

[9] Carlos FM Coimbra, Jan Kleissl, and Ricardo Marquez. Overview of solar-forecasting methods and a metric for accuracy evaluation-chapter 8. pages 171-194, 2013.
[10] Geoffrey E. Hinton Ronald J. Williams David E. Rumelhart. Learning representations by back-propagating errors. Nature, 323(533-536), 1986.

[11] Zibo Dong, Dazhi Yang, Thomas Reindl, and Wilfred M Walsh. Satellite image analysis and a hybrid esss/ann model to forecast solar irradiance in the tropics. Energy Conversion and Management, 79:66-73, 2014.

[12] Zibo Dong, Dazhi Yang, Thomas Reindl, and Wilfred M Walsh. A novel hybrid approach based on self-organizing maps, support vector regression and particle swarm optimization to forecast solar irradiance. Energy, 82:570-577, 2015.

[13] Jia-Hua Wei Fang-Fang Li, Si-Ya Wang. Long term rolling prediction model for solar radiation combining empirical mode decomposition (emd) and artificial neural network (ann)techniques. Renewable and Sustainable Energy, 10, 2018.

[14] Mahmoud Ghofrani, Mohadeseh Ghayekhloo, and Rasool Azimi. A novel soft computing framework for solar radiation forecasting. Applied Soft Computing, 48:207-216, 2016.

[15] X. Huang, J. Shi, B. Gao, Y. Tai, Z. Chen, and J. Zhang. Forecasting hourly solar irradiance using hybrid wavelet transformation and elman model in smart grid. IEEE Access, 7:139909-139923, 2019.

[16] Wu Ji and Keong Chan Chee. Prediction of hourly solar radiation using a novel hybrid model of arma and tdnn. Solar Energy, 85(5):808-817, 2011.

[17] Pedro F Jiménez-Pérez and Llanos Mora-López. Modeling and forecasting hourly global solar radiation using clustering and classification techniques. Solar Energy, 135:682-691, 2016.

[18] Amanpreet Kaur, Lukas Nonnenmacher, Hugo TC Pedro, and Carlos FM Coimbra. Benefits of solar forecasting for energy imbalance markets. Renewable Energy, 86:819-830, 2016.

[19] Stephane G Mallat. A theory for multiresolution signal decomposition: the wavelet representation. IEEE transactions on pattern analysis and machine intelligence, 11(7):674-693, 1989.

[20] Ricardo Marquez and Carlos FM Coimbra. Proposed metric for evaluation of solar forecasting models. Journal of solar energy engineering, 135(1):011016, 2013.

[21] Kasra Mohammadi, Shahaboddin Shamshirband, Chong Wen Tong, Muhammad Arif, Dalibor Petković, and Sudheer Ch. A new hybrid support vector machine-wavelet transform approach for estimation of horizontal global solar radiation. Energy Conversion and Management, 92:162-171, 2015.

[22] Stéphanie Monjoly, Maïna André, Rudy Calif, and Ted Soubdhan. Hourly forecasting of global solar radiation based on multiscale decomposition methods: A hybrid approach. Energy, 119:288-298, 2017.

[23] David Opitz and Richard Maclin. Popular ensemble methods: An empirical study. Journal of Artificial Intelligence Research, 11:169-198, 1999.

[24] Marius Paulescu, Eugenia Paulescu, Paul Gravila, and Viorel Badescu. Weather modeling and forecasting of PV systems operation. Springer Science \& Business Media, 2012.

[25] Ye Ren, PN Suganthan, and N Srikanth. Ensemble methods for wind and solar power forecasting - a state-of-the-art review. Renewable and Sustainable Energy Reviews, 50:82-91, 2015. 
[26] Saad Sayeef, Simon Heslop, David Cornforth, Tim Moore, Steven Percy, JK Ward, Adam Berry, and Daniel Rowe. Solar intermittency: Australia's clean energy challenge: characterising the effect of high penetration solar intermittency on australian electricity networks. CSIRO, Canberra, ACT, CSIRO Energy Transformed Flagship, 2012.

[27] Jürgen Schmidhuber Sepp Hochreiter. Long short-term memory. Neural Computation, 9(173-1780), 1997.

[28] Vishal Sharma, Dazhi Yang, Wilfred Walsh, and Thomas Reindl. Short term solar irradiance forecasting using a mixed wavelet neural network. Renewable Energy, 90:481-492, 2016.

[29] Nitikorn Silsirivanich. Fluctuation characteristics effect analysis of solar irradiance data by wavelet transform. 2017 AEDCEE, pages 301-306, 2017.

[30] Shikhar Srivastava and Stefan Lessmann. A comparative study of lstm neural networks in forecasting day-ahead global horizontal irradiance with satellite data. Solar Energy, 162:232-247, 2018.

[31] Marina Theodosiou. Forecasting monthly and quarterly time series using stl decomposition. International Journal of Forecasting, 27(4):1178-1195, 2011.

[32] Samuel R West, Daniel Rowe, Saad Sayeef, and Adam Berry. Short-term irradiance forecasting using skycams: motivation and development. Solar Energy, 110:188-207, 2014.

[33] Dazhi Yang, Vishal Sharma, Zhen Ye, Lihong Idris Lim, Lu Zhao, and Aloysius W Aryaputera. Forecasting of global horizontal irradiance by exponential smoothing, using decompositions. Energy, 81:111-119, 2015.

[34] Jie Zhang, Anthony Florita, Bri-Mathias Hodge, Siyuan Lu, Hendrik F Hamann, Venkat Banunarayanan, and Anna M Brockway. A suite of metrics for assessing the performance of solar power forecasting. Solar Energy, 111:157-175, 2015. 